\title{
A simple approach for describing metal-supported cyclohexaphenylene dehydrogenation
}

\section{Hybrid classical/DFT metadynamics simulations}

\author{
C.A. Pignedoli ${ }^{1}$, T. Laino ${ }^{2}$, M. Treier ${ }^{1, a}$, R. Fasel $^{1}$, and D. Passerone ${ }^{1, b}$ \\ 1 Empa, Swiss Federal Laboratories for Materials Testing and Research, nanotech@surfaces Laboratory, 8600 Dübendorf and \\ 3502 Thun, Switzerland \\ 2 IBM Zurich Research Laboratory, 8803 Rüschlikon, Switzerland
}

Received 7 November 2009

Published online 2 February 2010 - (C) EDP Sciences, Società Italiana di Fisica, Springer-Verlag 2010

\begin{abstract}
The mechanisms for the dehydrogenation reaction of cyclohexaphenylene at a copper surface are investigated with the help of density functional theory and metadynamics. Our results represent a showcase for an approach that describes the surface using many-body classical potentials and molecule-surface interactions with a van der Waals model. Starting from the experimental observation that dispersionassisted mechanisms are at least as important as catalytic processes for the description of the reaction, we fully describe the former, we identify intermediate states and estimate the free energy barriers that characterize the reaction.
\end{abstract}

\section{Introduction and experimental motivation}

The development of increasingly precise experimental techniques for the characterization and manipulation of molecules at surfaces has allowed an enormous development of the field of two-dimensional nanotechnology. The "bottom-up" realization of extended, self-assembled nanostructures with prescribed properties require a knowledge of the mechanisms leading to molecular adsorption on a certain substrate and to the formation of extended structures.

One particular field concerns the formation of carbonbased two-dimensional structures; the production of graphene single sheets and of graphene-like porous nanostructures starting from molecular precursors is becoming a research field per se $[1,2]$.

Recently, a procedure for the formation of graphene precursors has been proposed in our laboratory. Starting from cyclohexaphenylene (CHP, Fig. 3a), the first step is the multiple dehydrogenation of this molecule which is thus transformed into tribenzocoronene (TBC, Fig. 3f).

Experimentally, this process occurs at the $\mathrm{Cu}(111)$ surface at a temperature $T=470 \mathrm{~K}$ [3]. Figure 1 shows scanning tunneling microscopy (STM) images of different phases of the reaction, showing the occurrence of dimers and superstructures of intermediates.

\footnotetext{
a Present address: Institut de Science et d'Ingènierie Supramolèculaires, Université de Strasbourg 67000 Strasbourg, France.

b e-mail: daniele.passerone@empa.ch
}

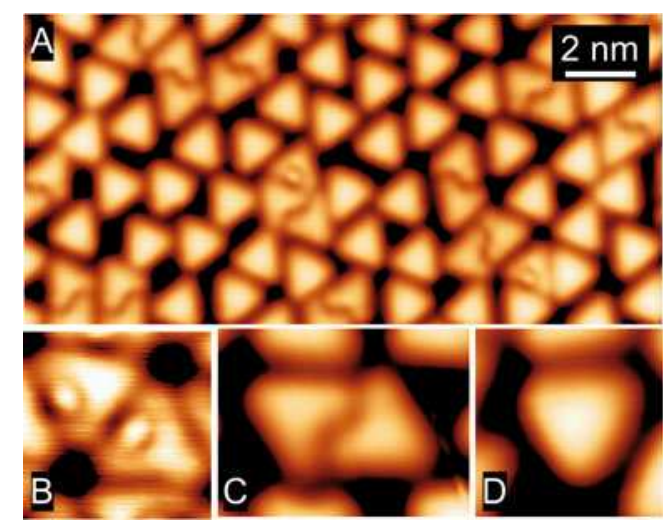

Fig. 1. (Color online) STM images of different phases of the reaction. (A) Sample annealed at $470 \mathrm{~K}$ showing mainly the final triangular TBC (scan parameters: $-0.6 \mathrm{~V} ; 50 \mathrm{pA}$ ). (B) Surface-stabilized reaction intermediates within self-assembled hexagonal superstructures $(-0.5 \mathrm{~V} ; 20 \mathrm{pA})$. (C) Dimer of second surface-stable reaction intermediates $(0.5 \mathrm{~V} ; 100 \mathrm{pA})$. (D) One fully reacted product molecule $(-0.6 \mathrm{~V} ; 50 \mathrm{pA})$. (A, C) and (D) have been recorded at $T=5 \mathrm{~K}$ while $\mathrm{B}$ was taken at $T=77 \mathrm{~K}$

An important question to be answered by modelers concerns the role of the surface: active substrates can influence the reaction mechanism by modifying the electronic structure of the adsorbate; relatively passive substrates, instead, not only play the role of a geometrical constraint for the adsorbed molecule, but can exchange heat with the adsorbate, and through dispersion forces contribute 
to enhance the planarity of specific molecule sections, thus affecting the reaction mechanisms.

Whereas chemistry-assisted mechanisms are often well encoded in organic chemistry literature, and it is possible to predict whether a certain process is likely to take place on the basis of chemical considerations, in presence of dispersion forces the conventional wisdom may not be sufficient to exclude the occurrence of a reaction channel that would be forbidden in ideal conditions (i.e., in the gas phase or in solution).

It is not easy to determine whether a certain reaction mechanism at the surface is dominated by a catalytic activity of the surface or by dispersive interactions. From the point of view of the modeler, this point is already crucial when choosing the level of theory to use in the simulations.

A full ab initio simulation of the whole system (surface plus adsorbate) is possible using density functional theory, and has become customary even for relatively extended structures (beyond 100 atoms) when the target is to obtain an equilibrium configuration or its electronic structure.

When the issue is dynamics, or even more, when rare events and free energies are concerned, some compromises must be made. A possibility is to limit the scope of the analysis to a certain class of processes, and leave to future work the task of extending it to other mechanisms.

By treating the molecule at a full or semi-empirical ab initio level, and the substrate as a classical metal interacting with the molecule through a Lennard-Jones like potential, we exclude chemical effects from our analysis. Such effects can however play a role, and have been discussed in another paper [3] on the basis of the experimental evidence and of additional calculations.

Here, we will focus on those reaction mechanisms assisted by the surface only through dispersive interactions. The goal of our analysis is to identify a reaction path that could explain the experimental findings: why ciclodehydrogenation proceeds only from $\mathrm{H}$ atoms of the "mobile" phenyl rings? Which are the intermediate products observed in the reactions? The selectivity of the dehydrogenation process suggests that surface catalysis cannot be the main mechanism characterizing the reaction. For this reason we focus here on the role played by van der Waals attractions in the reaction.

\section{Methods}

\subsection{Van der Waals interactions}

The main assumption in the present investigation is that no chemical bonding occurs between the molecule and the substrate. We focus on the role played by van der Waals interactions in driving the dehydrogenation reaction. The computational effort required by a standard density functional theory (DFT) approach with inclusion of dispersive force corrections (like e.g. Grimme [5]) on our system would not allow to investigate dynamical processes or a large variety of prototypical configurations. In order to be able to accurately describe the dynamics properties of the

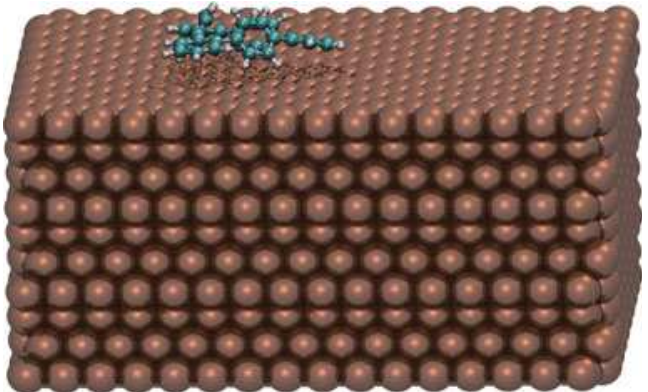

Fig. 2. (Color online) The simulation cell as described in the text, including 10 layers of $\mathrm{Cu}(111)$ and a CHP molecule adsorbed on it.

molecule/surface system and to investigate different intermediate geometries in the dehydrogenation process we designed a simple hybrid approach in which the molecule is treated within standard DFT or empirical DFT [6] (PM6) while the surface is described via embedded atom model potentials [7] (EAM). To include the van der Waals attraction we introduced a Molecule- $\mathrm{Cu}$ potential of the same form introduced by Levi and Calvini [8] for solving a problem of surface deformation under adsorption of organic molecules:

$$
V(r)=A_{v} \exp \left(-\alpha_{v} r\right)+A_{c} \exp \left(-\alpha_{c} r\right)-\frac{C_{6}}{r^{6}} .
$$

The first two terms take into account the repulsive energy arising from the Pauli principle, with contributions coming from the valence electrons and from the core electrons. The relative strength and decay lengths depend on the number of electrons and properties of the metal. For copper, following the discussion of Levi and Calvini [8], we choose $A_{c}=28 * A_{v}$. The $C_{6}$ parameters for the attractive part of the potential are deduced from a previous work by Grimme [5] while the repulsive part is fitted to ab initio calculations. The parameters $A_{x}$ and $\alpha_{x}$ (with $x=v, c$ ) are obtained through a fitting procedure as follows: we perform several ab initio simulations where the $\mathrm{Cu}(111)$ is modeled in a supercell containing $4 \mathrm{Cu}$ layers with 120 atoms each, and $20 \AA$ of vacuum, the empirical van der Waals correction to the PBE functional from Grimme [5] is used to include dispersive forces. We then compute the total energy for the CHP and for the TBC molecules displaced at different distances form the surface and fit the function (1) via the parameters $A_{v}, A_{c}, \alpha_{v}$ and $\alpha_{c}$. At the end of this procedure we fix the parameters to $C_{6}(\mathrm{C}-\mathrm{Cu})=75.41$ a.u., $C_{6}(\mathrm{C}-\mathrm{H})=21.33$ a.u., $A_{v}(\mathrm{C}-\mathrm{Cu})=4.136$ a.u., $A_{v}(\mathrm{C}-\mathrm{H})=0.878$ a.u., $\alpha_{v}=$ $1.33747 \mathrm{bohr}^{-1}$, and $\alpha_{c}=2.206825 \mathrm{bohr}^{-1}$.

Using these parameters we can setup the mixed DFTempirical simulation. The substrate is modeled through a slab of $2880 \mathrm{Cu}$ atoms (10 (111) layers) on which an isolated molecule is adsorbed (Fig. 2). Periodic boundary conditions are only applied to metal atoms, and the supercell, including a $30 \AA$ wide vacuum region above the surface, has an approximate size of $(40 \times 40 \times 70) \AA^{3}$. The restricted cell where the DFT calculations for the molecule are performed has a size of $30 \AA$ in all directions. 


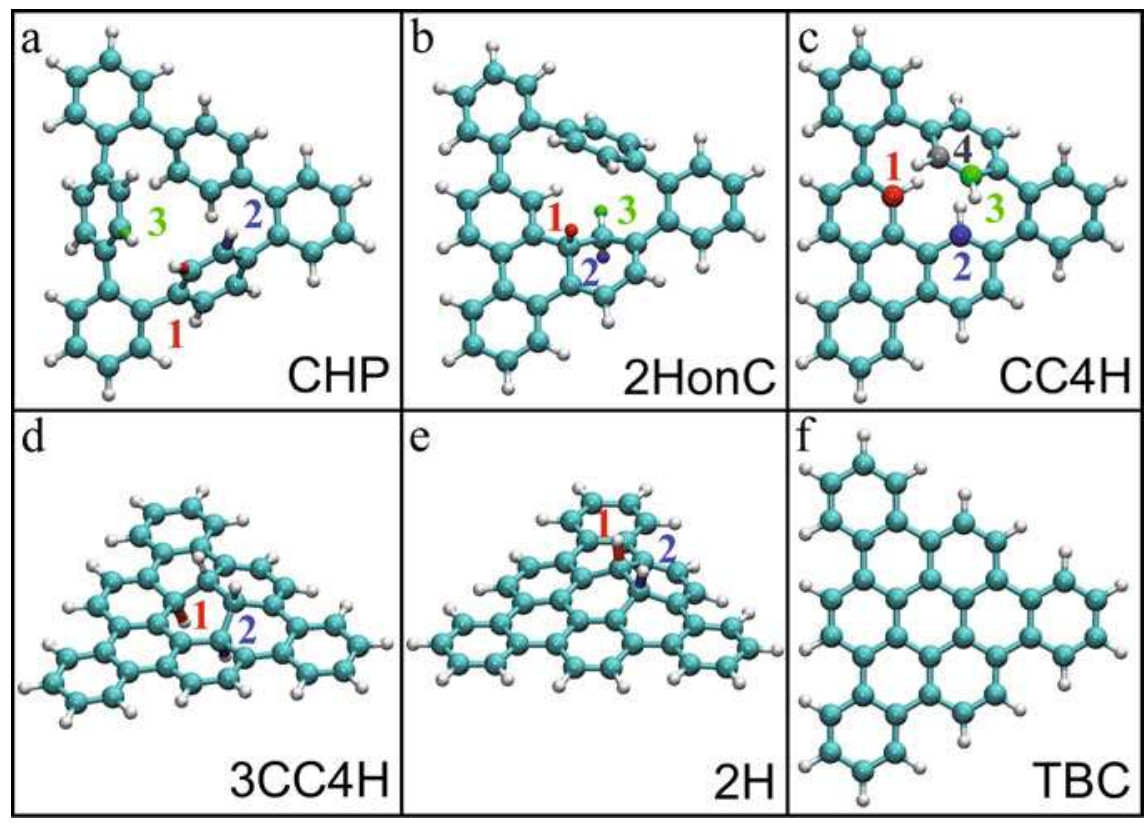

Fig. 3. (Color online) Initial and final states for the reaction steps described in the text.

$\mathrm{Cu}$ atoms interact via an embedded atom potential (EAM) [7] which involves a term dependent on the local atomic density. $\mathrm{Cu}$ interacts with the molecule through the potential described above, whereas $\mathrm{C}$ and $\mathrm{H}$ atoms in the molecule are described by standard DFT, with the BLYP exchange and correlation functional, GoedeckerTeter-Hutter norm-conserving pseudopotentials, within the Gaussian and plane waves (GPW) approach, which expands the wavefunction into a localized basis set and the charge density using Fourier transforms and thus plane waves. All calculations were done using the package cp2k [9]. Following the previous description, during a single step of dynamics or geometry optimization the metal atoms evolve due to pure classical forces (EAM potential + potential (1)) whereas the molecule atoms are subject to the classical potential (1) plus the DFT Hellmann-Feynman forces coming from the ionic and electronic degrees of freedom of the molecule.

The use of PM6, due to its extremely low computational cost, allowed us to investigate long dynamics trajectories and to identify intermediate states of the reaction. The most relevant geometries that arose from the trajectories were then refined with standard DFT [10].

\subsection{Metadynamics}

To estimate the free energy barriers that have to be overcome for the dehydrogenation reaction, we identified a possible set of intermediate steps and we used metadynamics [11] to explore in an unbiased manner the possible mechanisms of reaction.

Several methods to explore rare events through molecular dynamics simulations are available from the literature (see [12] and references therein). However, for such a complex reaction, none of them would be successful in finding the lowest energy path of the reaction in an unbiased way. Our goal is not finding the optimal reaction path but to give a case study of our simple method identifying possible reaction steps that are compatible with the experimental findings.

For our analysis we adopt the metadynamics method, which is based on a non-Markovian molecular dynamics simulation that allows to sample the free energy landscape spanned by a set of (typically two or three at most) collective variables whose combination is able to describe the mechanism under study.

The method has been successfully used to solve complex problems of different classes, ranging from biology to condensed matter physics and fundamental chemistry (see, e.g., [12] and references therein). Applications of metadynamics involving extended molecules and large barriers at a surface are instead not so common in literature [13], mainly because of the large size of the system and of the difficulty of choosing an appropriate and restricted set of collective variables.

The formulation adopted for the present problem is the so-called "direct metadynamics" [12]. A system is described by a set of coordinates $x$ (in the classical case, atomic positions) and a potential $V(x)$, evolving under the action of a thermostated Newtonian dynamics, which thus sample the canonical distribution at a temperature $T$.

Then, one is interested in studying the properties of the system as a function of a small number of collective variables $C_{\alpha}(x), \alpha=1, d$ (defined from now on as a vector $\boldsymbol{c}=\boldsymbol{C}(x)) d$ being 2 or 3 in practical applications. The collective variables can be any function of $x$, ranging from simple distances to coordination numbers or a radius of gyration of a polymer.

If the dynamics produced a very long time trajectory, the probability distribution of the chosen set of collective 
variables $\boldsymbol{C}$ could be extracted from the histogram of the collective variables along the trajectory. Then, the related free energy can be extracted from the negative logarithm of the related partition function times the temperature $T$.

Unfortunately, the trapping in metastable minima and the presence of high barriers in the potential energy surface hinder a complete sampling of the collective variable space. To overcome this problem, the dynamics is modified into an history-dependent random walk that starts from a free energy minimum and gradually explores regions of the configuration space of increasing size. To achieve the "escape" from the energy minima, a small repulsive Gaussian potential is added every $N_{G}$ MD steps (at intervals of time $\tau_{G}=N_{G} \tau$, being $\tau$ the time step of the MD), building up an external potential acting on the system at time $t$ :

$$
\begin{aligned}
& V_{G}(\boldsymbol{C}(x), t)= \\
& w \sum_{t^{\prime}<t, t^{\prime}=\tau_{G}, 2 \tau_{G}, \ldots} \exp \left(-\frac{\left(\boldsymbol{C}(x)-\boldsymbol{c}\left(t^{\prime}\right)\right)^{2}}{2 \delta s^{2}}\right),
\end{aligned}
$$

where the parameters are the Gaussian height $w$, its width $\delta s$, and the frequency $\tau_{G}$.

This potential fills the free energy landscape while the dynamics proceeds. This allows escaping deep energy minima and overcoming high barriers toward unexplored stable states.

In the limit of long times, the external potential $V_{G}$ can be proven to converge to an estimate of the underlying free energy, i.e., $F(\boldsymbol{c})=-\lim _{t \rightarrow \infty} V_{G}(\boldsymbol{C}(x), t)$.

Problems related to this method include:

- An inappropriate choice of the collective variable set; if the chosen collective variables do not describe completely the "slow" events happening in the process, a strong overestimate of the free energy and an hysteresis in the reconstruction of the free energy can occur.

- An insufficient sampling of the free energy space: the inverse of the potential (2) can represent a good approximation of the energy only if the resulting profile is sufficiently flat, and that implies a satisfactory sampling of the free energy space.

- The sampling of non-equilibrium high energy regions where the molecule can for example undergo undesired reactions like dissociations from which no recovery is possible.

The latter is exactly the case here. When discussing our results, we must remember that the system is not in thermodynamic equilibrium with a reservoir of hydrogen, corresponding to continuous dissociation and recombination at the molecule. Rather, when a hydrogen gets detached it will never attach back to the molecule; this corresponds to a situation where the hydrogen is continuously removed from the chamber (since it is done in ultra high vacuum).

Therefore, even if our metadynamics "suffers" from this lack of equilibrium as well (during the random walk in the collective variable space, hydrogen never comes back to the molecule) the predictions can be well compared with experiment concerning the forward barrier of reaction.
Instead, the free energy difference between minima and the backward reaction barriers are not correct as extracted from metadynamics, since the reverse process is not sampled. In order to correctly estimate the relative energies (at $T=0 \mathrm{~K}$ ) between the states, we resorted to local geometry optimizations of the different intermediates within standard DFT.

Since the barriers are quite high (in the order of $2 \mathrm{eV}$ ) we had to restrict the number of collective coordinates to three and we had to use multiple walkers to fill the free energy landscape (FES). Each metadynamics simulation was performed at $T=450 \mathrm{~K}$, a massive CSVR thermostat [14] was used and 50 ps dynamics of equilibration were performed for each initial state. One of the keys of our approach is the choice of different collective variables for the sequential steps of the reaction (see below).

\section{Results}

We identify five steps necessary to go from CHP to TBC;

Our simulation protocol is the following: first of all, the intermediate states are obtained starting from the initial states with successive metadynamics simulations ("escaping free energy minima" [12] using semiempirical PM6 DFT simulations. The single intermediate states found are then refined with standard DFT. The comparison of the intermediate structures with the experimental data will be published in another paper [3].

In a second step, our goal is to compute the free energy barriers between the intermediate steps by careful metadynamics sampling. For each step we use a different set of collective variables.

In Figure 3 we show the initial and final state for each step. We note that the intermediate states could have been located automatically through metadynamics, which can be used to escape free energy minima and find novel states. The insight provided by experiments suggests however that such intermediate states are configurations obtained by successive removal of two hydrogens.

\subsection{Reaction mechanisms}

\subsubsection{First step}

To estimate the FES of step 1 we used three distance collective variables $(\mathrm{CV})$ namely

$$
\begin{aligned}
& C V 1=d(H 1, C 2) \\
& C V 2=d(H 3, C 1) \\
& C V 3=d(C 1, C 3) .
\end{aligned}
$$

The first step goes from CHP to configuration $2 \mathrm{HonC}$, one $\mathrm{C}-\mathrm{C}$ bond is formed $(\mathrm{C} 1$ and $\mathrm{C} 3$ in panel $\mathbf{a}$, and two $\mathrm{H}$ atoms move in a concerted way to form a $\mathrm{CH}_{2}$ complex (as shown in panel a, $\mathrm{H} 1$ moves to $\mathrm{C} 2$ and $\mathrm{H} 3$ moves to $\mathrm{C} 1$ to obtain the geometry in panel $\mathbf{b}$ ). 

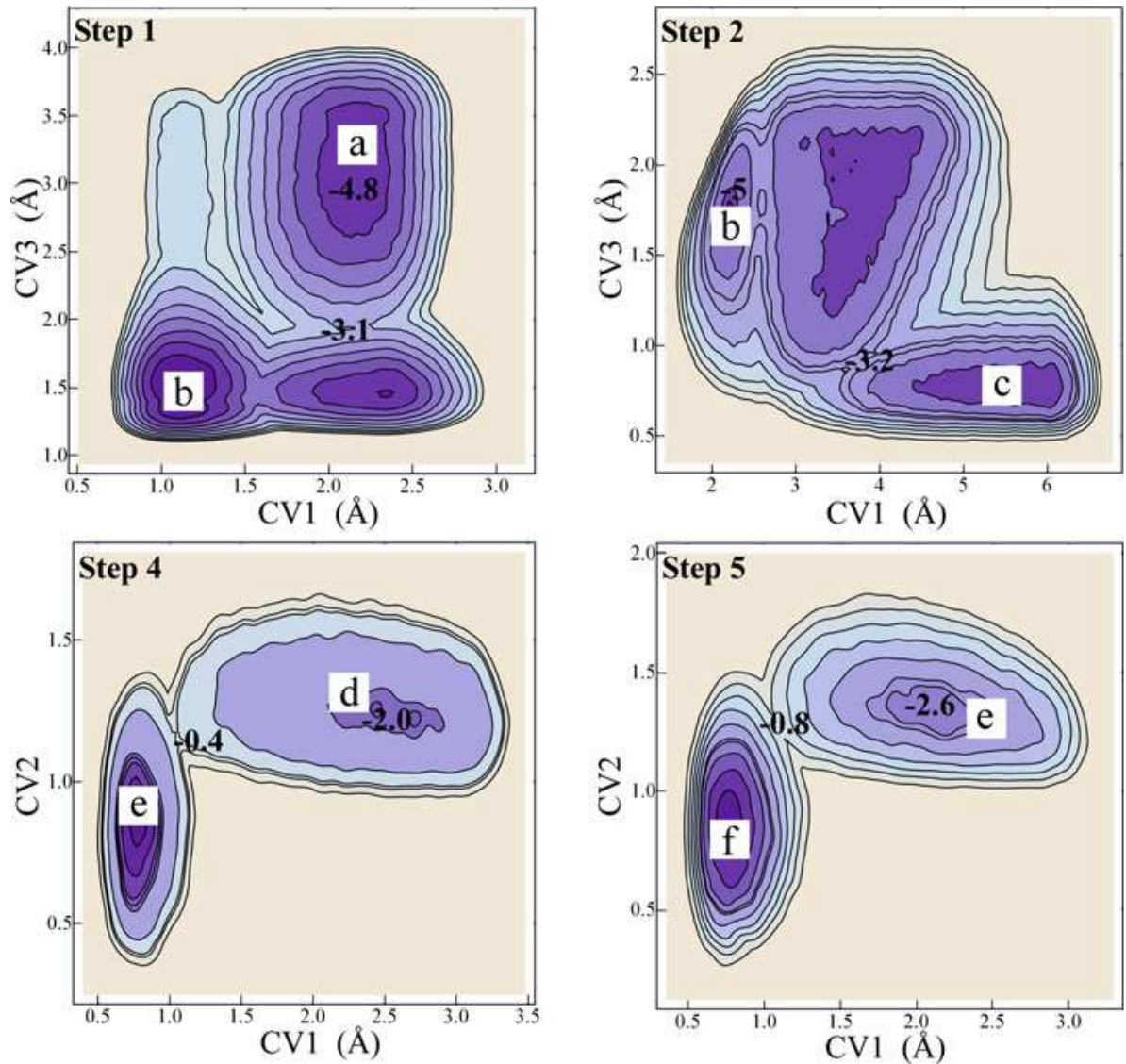

Fig. 4. (Color online) Free energy surfaces for the steps described in the text. One contour corresponds to $0.3 \mathrm{eV}$, and numbers (in $\mathrm{eV}$ ) correspond to free energies. The reliable barriers from the metadynamics simulations can be computed by subtraction of the numbers for transition and stable states.

This step is hindered by van der Waals attraction. To reach configuration $2 \mathrm{HonC} \mathrm{CHP}$ has to reduce its planarity. Hydrogen is not released and backward reaction has to be expected. This intermediate is not observed experimentally.

\subsubsection{Second step}

For step 2 we used the three following CVs:

$$
\begin{aligned}
& C V 1=d(H 2, C)+d(H 3, C) \\
& C V 2=d(H 1, C) \\
& C V 3=d(H 2, H 3) .
\end{aligned}
$$

In the second step the two $\mathrm{H}$ atoms of the complex $\mathrm{CH}_{2}$ detach from the molecule (with reference to panel $\mathbf{b}, \mathrm{H} 2$ and $\mathrm{H} 3$ detach from the common $\mathrm{C}$ atom) and a $\mathrm{H}$ atom attach to the $\mathrm{C}$ dangling bond ( $\mathrm{H} 1$ moves to the $\mathrm{C}$ atom common to $\mathrm{H} 2$ and $\mathrm{H} 3$ ). Eventually, configuration $\mathrm{CC} 4 \mathrm{H}$ is obtained. This step is strongly driven by van der Waals attraction: upon reaching configuration $\mathrm{CC} 4 \mathrm{H}$ the molecule becomes more planar and the system gains energy due to enhanced van der Waals attraction. All the subsequent steps are strongly driven by a gain in van der Waals energy due to increasing planarity of the molecule.

\subsubsection{Third step}

Step 3 is characterized by the formation of the remaining two $\mathrm{C}-\mathrm{C}$ bonds. No $\mathrm{H}$ is released during this step, and configuration $3 \mathrm{CC} 4 \mathrm{H}$ is obtained (as seen in panel c, bonding between $\mathrm{C} 1-\mathrm{C} 4$ and between $\mathrm{C} 2-\mathrm{C} 3$ brings to the configuration in panel $\mathbf{d}$ ).

Step 3 was investigated with a 1D metadynamics simulation. We used as a collective variable $C V 1=d(C 1, C 4)+$ $d(C 2, C 3)$.

In this third step, the role of the surface is again to increase the planarity of the molecule.

\subsubsection{Fourth and fifth step}

For step 4 and for step 5 we used

$$
\begin{aligned}
& C V 1=d(H 1, H 2) \\
& C V 2=c / d(C 2, H 1) \\
& C V 3=c / d(C 1, H 2)+c / d(C 2, H 1) .
\end{aligned}
$$

The last two steps are the successive release of two $\mathrm{H}_{2}$ molecules forming the $2 \mathrm{H}$ intermediate (panel e) and the final state (panel f).

In Figure 4 we show 2D cuts of the 3D FES for steps 1, $2,4,5$. 
Table 1. Relative optimized energies and free energy barriers for the reaction steps described in the text.

\begin{tabular}{l|cccccc}
\hline & $\mathbf{a}$ & $\mathbf{b}$ & $\mathbf{c}$ & $\mathbf{d}$ & $\mathbf{e}$ & $\mathbf{f}$ \\
\hline Total energy relative to f $(\mathrm{eV})$ & 1.84 & 2.43 & 1.50 & 2.88 & 1.88 & \\
Free energy barrier [step] $(\mathrm{eV})$ & & $1.7[1]$ & $1.8[2]$ & $1.8[3]$ & $1.6[4]$ & $1.8[5]$ \\
\hline
\end{tabular}

\subsection{Relative energies and free energy barriers}

In Table 1 we present the relative energies of all the above structures assuming TBC as reference. The energy of a H2 molecule was used to define the hydrogen chemical potential in comparing structures with different numbers of $\mathrm{H}$ atoms. An estimate of the free energy surface (FES) barriers is also included in the table. As already discussed, metadynamics allows to estimate the forward reaction barriers for this process at the experimental temperature, whereas the intermediate energies are computed in standard DFT with local optimizations at $T=0 \mathrm{~K}$.

The barriers height that we obtain are compatible with the temperature and time scale of the experiment and are lower compared to the energy required to dehydrogenate a phenyl ring on $\mathrm{Cu}(111)$ (2.2 eV, see e.g. [4]) Steps 2, 4 and 5 are irreversible due to $\mathrm{H}$ removal from the chamber. Only intermediates 3 and 5 should be observed experimentally.

\section{Conclusions}

In this paper we have shown that the use of a simple model for molecule/substrate interactions, coupled with metadynamics simulations can shed light on the possible reaction mechanisms for the dehydrogenation of aromatic molecules adsorbed on metals.

Although the description of such mechanisms is not complete, since catalytic effects of copper can not be taken into account at this level of theory, our results show that already the steric hindrance and the van der Waals attraction make reaction mechanisms that would be forbidden in the gas phase possible at the experimental temperatures and in presence of the metal surface.

Such mechanisms are competitive with other involving electronic structure modifications at the surface, as it will be shown in a forthcoming paper. We think that the combination of metadynamics with a model that allows a description of the complete system albeit at a simplified level concerning certain details of the physics involved, represents a powerful tool for the investigation of the increasingly important class of problems that concern self-assembly at surfaces.

Computing time at the Swiss Supercomputing Center (CSCS) in Manno is gratefully acknowledged. It is a pleasure to dedicate the present paper to Andrea Levi on occasion of his retirement.

\section{References}

1. L.J. Zhi, K. Müllen, J. Mater. Chem. 18, 1472 (2008)

2. D.F. Perepichka, F. Rosei, Science 323, 216 (2009)

3. M. Treier, C.A. Pignedoli, T. Laino, R. Rieger, K. Müllen, D. Passerone, R. Fasel, paper in preparation

4. S.J. Jenkins, Proc. R. Soc. A 465, 2949 (2009)

5. S. Grimme, J. Comput. Chem. 27, 1787 (2006)

6. J.J.P. Stewart, Journal of Molecular Modeling 13, 1173 (2007)

7. S.M. Foiles, M.I. Baskes, M.S. Daw, Phys. Rev. B 33, 7983 (1986)

8. A.C. Levi, P. Calvini, Surf. Sci. 601, 1494 (2007)

9. http://cp2k.berlios.de

10. A.D. Becke, Phys. Rev. A 38, 3098 (1988)

11. A. Laio, M. Parrinello, Proc. Natl. Acad. Sci. U. S. A. 99, $12562(2002)$

12. A. Laio, F. Gervasio, Rep. Prog. Phys. 71, 126601 (2008)

13. A. Rodriguez-Fortea, M. Iannuzzi, J. Phys. Chem. C 112, $19642(2008)$

14. G. Bussi, D. Donadio, M. Parrinello, J. Chem. Phys. 126, 014101 (2007) 